\title{
CRYOGENIC TREATMENT OF HERPES SIMPLEX KERATITIS*
}

BY

TADEUSZ KRWAWICZ

Ophthalmological Clinic, Medical Academy, Lublin, Poland

DURING the early stage of our experiments on the use of low temperature for cataract extraction (Krwawicz, 1961, 1963), we had several opportunities of observing the effects of accidentally touching the cornea with the cryoextractor refrigerated to about $-79^{\circ} \mathrm{C}$. This accident proved to be quite harmless in all cases as no lasting effect on the clarity of the cornea could be seen after the tissue had been separated from the instrument. The ability of the cornea to return to its normal condition after coming into contact with the refrigerated metal suggested the possibility of the therapeutic application of low temperature to the cornea itself.

A series of experiments on animal eyes was then carried out, and it was found that when the cornea was touched with an appropriate instrument refrigerated to $-79^{\circ} \mathrm{C}$., there occurred an immediate freezing of the epithelium and superficial layers of the corneal stroma. Repeated refrigeration of a delimited area of corneal tissue had no ill effects on its transparency and did not produce any noteworthy fall in the temperature measured in the anterior chamber (Krwawicz, 1964).

This cryogenic procedure was then used to treat corneal lesions in patients suffering from herpes simplex keratitis, with highly satisfactory results.

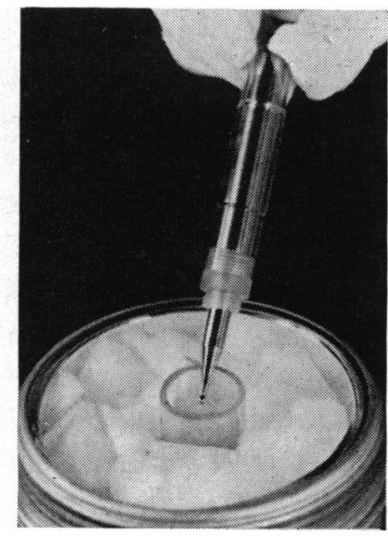

FIG. 1.-Cryoapplicator removed from refrigerating mixture, ready for use.
Technique

Initially, the instrument used for these corneal applications was very similar to the cryoextractor, but it has recently been modified so that the conical part is thicker, there is no division into steps, and the ball-shaped end measures at least $2 \mathrm{~mm}$. in diameter (Fig. 1). The cryoapplicator is refrigerated in the same way as the cryoextractor, i.e. in a mixture of dry ice $\left(\mathrm{CO}_{2}\right)$ and methyl alcohol, but the ball-shaped end is not coated with ice, and there is no need to insulate the conical part with a silk thread.

Under local anaesthesia, a speculum is inserted and the eyeball is fixed with fixation forceps. The end of the refrigerated instrument is then applied to the margin of the ulceration previously stained with fluorescein. An attempt is then made to cover with a few touches the whole ulceration, i.e. its centre and all its ramifications with their nearest surroundings. Each 
application, not exceeding ten in number, lasts a few seconds, and between the applications the ball of the instrument is wiped with a sterile napkin to remove the hoar-frost. This momentary and circumscribed application of low temperature to the cornea produces a transient freezing of the epithelium and superficial layers of the stroma; the whitish area of the partially frozen tissue clears up immediately after the removal of the cryoapplicator and regains its former transparency (Figs 2 and 3).

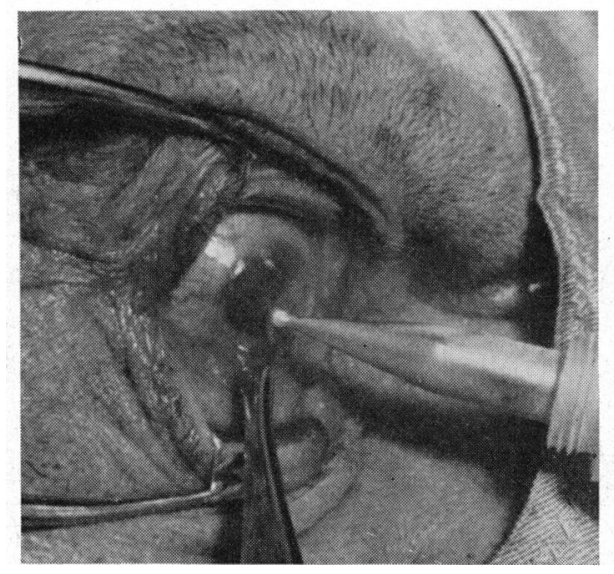

FIG. 2.-The margin of the ulceration is touched with the ball-shaped end of the cryoapplicator.

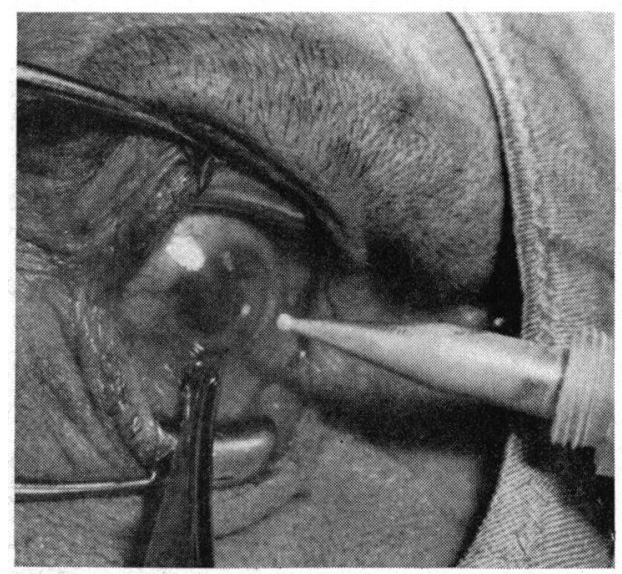

Fig. 3.-A round whitish spot of local refrigeration, corresponding in size to the end of the instrument, is seen in the cornea after the removal of the cryoapplicator, but these spots swiftly return to normal.

The operation is followed by the administration of antibiotics into the conjunctival sac to prevent secondary infection.

\section{Results}

In most cases one treatment is enough to obtain healing of the herpetic lesions, but if the improvement is only temporary, or there is no improvement at all, the application may be repeated after a few days' interval. If necessary, more than one further application may be made. Sometimes a certain spread of the lesions is observed on the day after the operation, but this regresses very quickly.

In an initial series of 41 patients affected with herpes simplex keratitis (Krwawicz, 1964) cryoapplication gave uniformly good results. The patients were regarded as cured when the ulceration ceased to take the fluorescein stain, although a superficial clouding or characteristic dendritic shadow sometimes remained.

In patients with stromal involvement the average duration of the treatment was 8.8 days, but when the lesions were superficial, healing was obtained in about $3 \cdot 2$ days. One patient had a recurrence within one month but the long-term results, extending over more than a year, which are now available for this group of patients, demonstrate that the cure has been permanent.

Cryoapplication has recently been used in a further twelve patients with undoubtedly herpetic corneal lesions. It was found that the instrument could be applied to the cornea for up to 7 seconds without any risk of complication, and the average duration of treatment was reduced to 6.2 days in cases with stromal involvement, and to 2.8 days with superficial lesions. 


\section{Discussion}

These results show that direct, momentary application of the refrigerated instrument to the area of corneal ulceration of herpetic origin assists the healing of the lesions.

Speedy healing was obtained in patients who had been previously treated by other methods without success, and in no case was it necessary to interrupt the cryogenic therapy because of poor response or harmful side-effects. In most cases the site of the ulcer cleared up, and there remained only a scar, more or less delicate, according to the depth of the lesion.

Reports on the use of 5-iodo-2'-deoxyuridine (IDU) in herpes simplex keratitis (Kaufman, 1962; Kaufman, Martola, and Dohlman, 1962) suggest that rapid changes are to be expected in this field of ocular therapy, but IDU is ineffective in certain cases (Luntz and MacCallum, 1963; Maxwell, 1963), depending on the degree of involvement of the corneal stroma. When the lesions are superficial the results may be excellent, but in cases with deeper stromal involvement they are not so good.

Low temperature therapy has been used in our clinic since the end of 1962, and although the number of cases treated is by no means abundant, we feel that our results deserve attention. In our experience this method has been consistently successful; it is also simple and free from risk, and does not inconvenience the patient.

\section{Summary}

Herpes simplex keratitis may be treated by the application of low temperature. The cryoapplicator, refrigerated to $-79^{\circ} \mathrm{C}$., is applied to the corneal lesion several times for up to 7 seconds each time. A good result was obtained in all patients so far treated by this method.

\section{REFERENCES}

Kaufman, H. E. (1962). Proc. Soc. exp. Biol. (N.Y.), 109, 251.

-, Martola, E. L., and Dohlman, C. (1962). Arch. Ophthal., 68, 235.

KRWAWICZ, T. (1961). Brit. J. Ophthal., 45, 279.

(1963). Ibid., 47, 36.

- (1964). Klin. oczna, 34, 435.

Luntz, M. H., and MacCallum, F. O. (1963). Brit. J. Ophthal., 47, 449.

MAXWell, E. (1963). Amer. J. Ophthal., 56, 571. 